Bull. Austral. Math. Soc.

$20 \mathrm{c} 15,20 \mathrm{c} 20$

VOL. $62(2000) \quad[525-528]$

\title{
A SUBMATRIX OF THE CHARACTER TABLE
}

\section{GABRIEL NAVARRO}

Let $G$ be a finite group and let $p$ be a prime number. We consider the submatrix of the character table of $G$ whose rows are indexed by the characters in blocks of maximal defect, and whose columns are indexed by the conjugacy classes of $p^{\prime}$-size. We prove that this matrix has maximum rank.

\section{INTRODUCTION}

Let $G$ be a finite group and let $p$ be a prime number. Our aim in this note is to show the following result.

Theorem A. Suppose that $P$ is a Sylow $p$-subgroup of $G$. Let $\mathcal{X}$ be a set of representatives of the conjugacy classes of $G$ with defect group $P$ and let $\mathcal{Y}$ be the set of irreducible characters of $G$ in blocks with defect group $P$. Then $|\mathcal{X}| \leqslant|\mathcal{Y}|$ and the matrix

$$
(\chi(x))_{\chi \in \mathcal{Y}, x \in \mathcal{X}}
$$

has rank $|\mathcal{X}|$.

We notice that Theorem A is not longer true if we replace the Sylow $p$-subgroup $P$ of $G$ by any $p$-subgroup of $G$. (For instance, that would imply that any group with an element $x \in G$ such that $\left|\mathrm{C}_{G}(x)\right|$ is not divisible by $p$ has a $p$-defect zero character, and this is simply false.) We also remark that the sets $\mathcal{Y}$ and $\mathcal{X}$ can easily be identified by the character table, so we can view Theorem $\mathrm{A}$ as another application of modular representation theory to ordinary character theory.

Corollary B. Let $G$ be a finite group and let $P$ be a Sylow $p$-subgroup of $G$. Assume that $x, y \in G$ are such that $p$ does not divide $\left|G: \mathbf{C}_{G}(x)\right|$ and $\left|G: \mathbf{C}_{G}(y)\right|$. Then $x, y$ are $G$-conjugate if and only if $\chi(x)=\chi(y)$ for all $\chi \in \operatorname{Irr}(G)$ in blocks with defect group $P$.

If $x, y \in G$ are $p$-elements, Corollary B follows, of course, from block orthogonality (although our methods here are more elementary).

Finally, we would like to mention that this note began as an attempt to understand what we now believe to be a fact: in a group $G$ with an Abelian Sylow $p$-subgroup, the

Received 22nd May, 2000

Research partially supported by DGICYT.

Copyright Clearance Centre, Inc. Serial-fee code: 0004-9727/00 \$A2.00+0.00. 
submatrix of the character table of $G$ formed by the characters of degree not divisible by $p$ and the elements whose conjugacy class size is not divisible by $p$ has maximum rank. This result would be a consequence of Theorem $A$ and the height zero conjecture.

\section{PROOFS}

We let $R$ be the ring of algebraic integers in $\mathbb{C}$. We fix a prime $p$ and we choose a maximal ideal $M$ of $\mathbf{R}$ containing $p \mathbf{R}$. Let $F=\mathbf{R} / M$ and let

$$
\text { * }: \mathbf{R} \rightarrow F
$$

be the natural ring homomorphism. Let

$$
S=\left\{\frac{r}{s} \mid r \in \mathbf{R}, s \in \mathbf{R}-M\right\},
$$

a local ring. Then

$$
\mathbf{J}(S)=\left\{\frac{r}{s} \mid r \in M, s \in \mathbf{R}-M\right\}
$$

is the Jacobson radical of $S$. Of course, the map ${ }^{*}: \mathbf{R} \rightarrow F$ can be extended to $S$ in a natural way. We follow the notation in [1].

Recall that the conjugacy class of $x \in G$ has defect group $P$ if some $G$-conjugate of $P$ is in $\operatorname{Syl}_{p}\left(\mathbf{C}_{G}(x)\right)$.

Proof of Theorem A: Let $N=\mathrm{N}_{G}(P)$ and $C=\mathrm{C}_{G}(P)$. If $H$ is a group, $R$ is a ring, and $X \subseteq H$ we denote by

$$
\widehat{X}=\sum_{x \in X} x \in R H .
$$

We define

$$
\alpha: \mathbf{Z}(S G) \rightarrow \mathbf{Z}(S N)
$$

by setting $\alpha(\widehat{K})=\widehat{K \cap C}$ for $K \in \operatorname{cl}(G)$, and extending $S$-linearly (where it is understood that $\alpha(\widehat{K})=0$ if $K \cap C=\emptyset)$.

Consider ${ }^{*}: \mathbf{Z}(S G) \rightarrow \mathbf{Z}(F G)$ given by

$$
\left(\sum_{K \in \operatorname{cl}(G)} a_{K} \widehat{K}\right)^{*}=\sum_{K \in \operatorname{cl}(G)}\left(a_{K}\right)^{*} \widehat{K}
$$

This is a ring homomorphism with kernel $\mathbf{J}(S) \mathbf{Z}(S G)$. Also let us denote by * : $\mathbf{Z}(S N) \rightarrow \mathbf{Z}(F N)$ the corresponding ring homomorphism for $N$. If $\operatorname{Br}_{P}: \mathbf{Z}(F G) \rightarrow$ $\mathbf{Z}(F N)$ is the Brauer homomorphism (see $[1, \mathrm{p} .85]$ ), we have that

$$
\operatorname{Br}_{P}\left(x^{*}\right)=\alpha(x)^{*}
$$


for $x \in \mathbf{Z}(S G)$.

If $\chi \in \operatorname{Irr}(G)$, then we denote by $e_{\chi}$ the character idempotent. If $B$ is a $p$-block of $G$, then we write $f_{B}=\sum_{\chi \in \operatorname{Irr}(B)} e_{\chi}$ (see $\left.[1, \mathrm{p} .51]\right)$. Let us denote by $\operatorname{Bl}(G \mid P)$ the set of blocks of $G$ such that $P$ is a defect group of $B$. Now, let

$$
u=\sum_{B \in \mathrm{BI}(G \mid P)} f_{B} \in \mathbf{Z}(S G)
$$

(by $[1$, Corollary 3.8$])$, and let

$$
v=1-u=\sum_{B \in \mathrm{B} \mid(G)-\mathrm{Bl}(G \mid P)} f_{B} .
$$

We have that $\mathbf{Z}(S G)=U \oplus V$, as $S$-modules, where $U=\mathbf{Z}(S G) u$ and $V=\mathbf{Z}(S G) v$.

Now, we claim that $\operatorname{Br}_{P}\left(V^{*}\right)=0$. Since the Brauer homomorphism and * are ring homomorphisms, it is enough to show that if $B \in \mathrm{Bl}(G)-\mathrm{Bl}(G \mid P)$, then $\operatorname{Br}_{P}\left(e_{B}\right)=$ 0 , where $e_{B}=\left(f_{B}\right)^{*}$. Since $P \in \operatorname{Syl}_{p}(G)$, this follows from [1, Theorem (4.11)]. Now, we have that

$$
\alpha(\mathbf{Z}(S G))^{*}=\operatorname{Br}_{P}\left(U^{*}+V^{*}\right)=\operatorname{Br}_{P}\left(U^{*}\right)=\alpha(U)^{*} .
$$

Now, given $\sum_{K \in \mathrm{cl}(G)} a_{K} \widehat{K} \in \mathbf{Z}(S G)$, there exists $\sum_{K \in \mathrm{cl}(G)} b_{K} \widehat{K} \in U$ such that

$$
\sum_{K \in \mathrm{cl}(G)}\left(a_{K}\right)^{*} \widehat{K \cap C}=\sum_{K \in \mathrm{cl}(G)}\left(b_{K}\right)^{*} \widehat{K \cap C} .
$$

Hence $a_{K}-b_{K} \in \mathbf{J}(S)$ whenever $K \cap C$ is not empty. Hence

$$
\begin{aligned}
\alpha\left(\sum_{K \in \operatorname{cl}(G)} a_{K} \widehat{K}\right)-\alpha\left(\sum_{K \in \mathrm{cl}(G)} b_{K} \widehat{K}\right) & \\
= & \sum_{\substack{K \in \mathrm{cl}(G) \\
K \cap C \neq \emptyset}}\left(a_{K}-b_{K}\right) \widehat{K \cap C} \in \mathbf{J}(S) \alpha(\mathbf{Z}(S G)) .
\end{aligned}
$$

Therefore

$$
\alpha(\mathbf{Z}(S G))=\alpha(U)+\mathbf{J}(S) \alpha(\mathbf{Z}(S G))
$$

Now, we have that $\alpha(\mathrm{Z}(S G))$ is a finitely generated $S$-module and that $\alpha(U)$ is an $S$-submodule of $\alpha(\mathrm{Z}(S G))$. By Nakayama's Lemma [1, Lemma (1.7)], we conclude that

$$
\alpha(U)=\alpha(\mathbf{Z}(S G))
$$


Now, let $\beta: \mathbf{Z}(\mathbb{C} G) \rightarrow \mathbf{Z}(\mathbb{C} N)$ be the map $\beta(\widehat{K})=\widehat{K \cap C}$ for $K \in \mathbf{c l}(G)$, extended C-linearly.

Let us denote by $\operatorname{cl}(G \mid P)$ the set of conjugacy classes of $G$ with defect group $P$. If $K$ is a conjugacy class of $G$, note that $K \cap C \neq \emptyset$ if and only if $K$ has defect group $P$ (because $P$ is a Sylow $p$-subgroup of $G$ ). Also, we have that the map $K \mapsto K \cap C$ is a bijection $\operatorname{cl}(G \mid P) \rightarrow \operatorname{cl}(N \mid P)$ by $[1$, Lemma (4.16)]). We have that

$$
\beta(\mathbf{Z}(\mathbb{C} G) u)=\mathbf{Z}_{P}(\mathbb{C} N)
$$

where $\mathbf{Z}_{P}(\mathbb{C} N)$ is the $\mathbb{C}$-span of the conjugacy classes of $N$ with defect group $P$. Now,

$$
\mathbf{Z}(\mathbb{C} G) u=\sum_{\chi \in \mathcal{Y}} \mathbf{Z}(\mathbb{C} G) e_{\chi}
$$

and we have that $\left\{e_{\chi} \mid \chi \in \mathcal{Y}\right\}$ is a $\mathbb{C}$-basis of $\mathbf{Z}(\mathbb{C} G) u$ (recall that the character idempotents are orthogonal, and therefore, linearly independent). Now,

$$
\beta\left(e_{\chi}\right)=\frac{\chi(1)}{|G|} \sum_{K \cap C \neq \emptyset} \overline{\chi\left(x_{K}\right)} \widehat{K \cap C}
$$

and it follows that the matrix of the surjective linear map $\beta_{\mathbf{Z}(\mathbb{C} G) u}$ with respect to the bases

$$
\left\{e_{\chi} \mid \chi \in \mathcal{Y}\right\} \quad \text { and } \quad\{\widehat{K \cap C} \mid K \in \operatorname{cl}(G \mid P)\}
$$

has the same rank as the matrix in the statement of the theorem.

Of course, Corollary B follows from Theorem A since we cannot have two identical columns in a full rank matrix having no more columns than rows.

\section{REFERENCES}

[1] G. Navarro, Characters and blocks of finite groups, London Mathematical Society Lecture Note Series 250 (Cambridge University Press, Cambridge, 1998).

Departament d'Àlgebra

Facultat de Matemàtiques

Universitat de València

46100 Burjassot

València

Spain

e-mail: gabriel@uv.es 